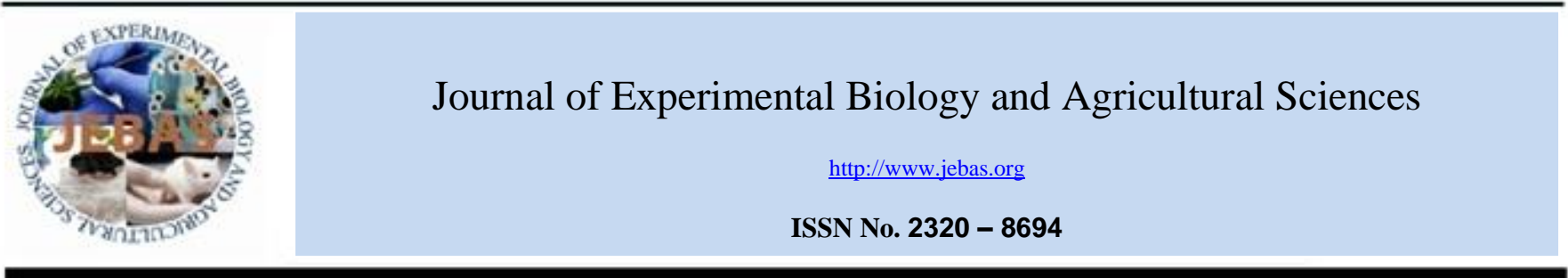

\title{
MICROFILARAEMIA INDUCED CORNEAL OPACITY IN A WATER BUFFALO (Bubalus bubalis): A CASE REPORT AND REVIEW OF LITERATURE
}

\author{
Khan Sharun ${ }^{1 *}$, Manjusha K. M. ${ }^{1}$, Aswathy Gopinathan ${ }^{1}$, Kiranjeet $\operatorname{Singh}^{1}$, Bindu Lakshmanan ${ }^{2}$, \\ Naveen Kumar Verma ${ }^{1}$, Kuldeep Dhama ${ }^{3 *}$, A. M. Pawde ${ }^{1}$
}

${ }^{1}$ Division of Surgery, ICAR-Indian Veterinary Research Institute, Izatnagar, Bareilly, Uttar Pradesh

${ }^{2}$ Department of Veterinary Parasitology, Kerala Veterinary and Animal Sciences University, Pookode, Kerala.

${ }^{3}$ Division of Pathology, ICAR-Indian Veterinary Research Institute, Izatnagar, Bareilly, Uttar Pradesh.

Received - January 11, 2020; Revision - February 15, 2020; Accepted - February 22, 2020

Available Online - February 25, 2020

DOI: http://dx.doi.org/10.18006/2020.8(1).9.14

\author{
KEYWORDS \\ Microfilaraemia \\ Setaria labiatopapillosa \\ Corneal opacity \\ Water buffalo \\ Bubalus bubalis
}

* Corresponding author

E-mail: sharunkhansk@gmail.com (Khan Sharun); kdhama@ rediffmail.com (Kuldeep Dhama)

Peer review under responsibility of Journal of Experimental Biology and Agricultural Sciences.

Production and Hosting by Horizon Publisher India [HPI] (http://www.horizonpublisherindia.in/).

All rights reserved.

\begin{abstract}
Corneal opacity is the common manifestation of several infectious and non-infectious aetiologies. Systematic diagnostic investigation is necessary to identify the reason behind such clinical sign. An adult pregnant water buffalo (Bubalus bubalis) was presented with the history of fever, anorexia, and corneal opacity. The animal was found to be lethargic, weak and anaemic on clinical examination. Ophthalmological examination identified marked bilateral corneal opacity. Haematological investigation revealed severe anaemia and leukocytosis characterized by neutrophilia, lymphocytopenia, and monocytosis. Examination of peripheral wet film mount identified the presence of moving parasites which was later identified to be sheathed microfilaria. Based on micrometric analysis, it was identified as the microfilaria of Setaria labiatopapillosa. Hence the case was confirmed as bubaline microfilariosis. The animal was treated with subcutaneous administration of ivermectin at $200 \mu \mathrm{g} / \mathrm{kg}$ body weight along with other supportive therapies. The animal succumbed to death later that day before performing any further investigation. The purpose of this case report is to put on records an unusual and rare case of corneal opacity secondary to microfilaraemia due to S. labiatopapillosa in a water buffalo. Microfilariosis can lead to severe consequences in case of delayed presentation, diagnosis, and treatment. It can cause severe forms of anaemia that can be highly fatal if not corrected. The extensive analysis performed in this paper will act as a guideline for the differential diagnosis of cases presented with corneal opacity in bovines. Rapid diagnosis and treatment can significantly improve the production and health status of the animals.
\end{abstract}

All the articles published by Journal of Experimental Biology and Agricultural Sciences are licensed under a Creative Commons Attribution-NonCommercial 4.0 International License Based on a work at www.jebas.org.

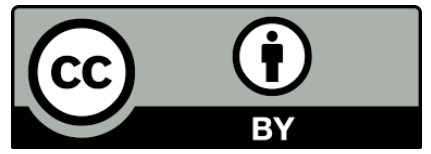




\section{Introduction}

Filariosis is caused by the helminths belonging to the superfamily Filaroidea. The parasites belonging to the genera Setaria, Onchocerca, Stephanofilaria, and Parafilaria are the causative agents of filariosis in cattle and buffalo. The latter three genera (except Onchocerca armillata), produce cutaneous lesions and hence their microfilariae/larval stage are not found in the blood circulation (Satish, 1996). Bubaline microfilariosis is a vector borne disease caused by the larvae of Setaria cervi, S. digitata, $S$. labiatopapillosa, and $O$. armillata. The larvae of these worms are called as microfilariae and they circulate in the peripheral blood (Rani et al., 2009; Sundar \& D'Souza, 2015). The filarial nematodes of Setaria sp. are commonly found in peritoneal cavity of the cattle. Although adult worms are considered to be nonpathogenic, mild fibrinous peritonitis may occur sporadically. The erratic migration of the larval forms can cause serious conditions in animals (Sundar \& D'Souza, 2015).

Microfilariosis was found to be associated with gradual deterioration in the body condition of the animals. Farmers suffer economic loss occurring due to decline in milk yield, cost of treatment and the mortality of affected animals (Samatha et al., 2016). Jayalakshmi et al. (2017) reported a prevalence of $2.72 \%$ in bovines, while in buffalo it was recorded as $2.97 \%$ and $2.45 \%$ in cattle. The higher prevalence of microfilariosis in buffaloes can be due to their habitat (swampy areas) which provides a suitable environment for vector population. Seasonal variation was also observed in the prevalence rate of microfilariosis. Highest prevalence was observed in monsoon season, followed by summer, and least in the winter season (Samatha et al., 2016). The prevalence of microfilariosis was found to be higher in lactating animals compared to heifers and non-lactating animals. The higher susceptibility of lactating animals can be attributed to the role played by the prolactin hormone in producing immunosuppression along with the lactation stress (Jayalakshmi et al. 2017).

The main clinical manifestations associated with microfilariosis are anaemia, debility, loss of appetite, oedematous swelling (mostly in the abdominal region), increased respiration rate, and heart rate (Sharma \& Joshi, 2002). Diagnosis of microfilariosis can be done by using diagnostic techniques like wet mount examination, Giemsa stained blood smear, Modified Knott's method, and Citrate-Saponin-acid method. Among these, CitrateSaponin-acid method was found to be the most efficient and sensitive diagnostic tool for the detection of microfilaraemia (Bino Sundar \& Ravindran, 2010). Even though wet mount examination has low sensitivity, it can be used for initial screening due to simplicity in its procedure. Haematological changes observed in microfilariosis include decrease in the haemoglobin concentration, total erythrocyte count, and packed cell volume leading to anaemia. There can also be an increase in the total leukocyte count due to the relative increase in lymphocyte and eosinophil counts (Sharma \& Joshi, 2002).

Corneal opacity is a clinical manifestation associated with several parasitic and infectious diseases in cattle and buffalo. It is seen associated with parasitic diseases due to Setaria digitata, Thelazia sp., Theileria annulata, Trypanosoma evansi, and Plasmodium bubalis (Mohan et al., 2009; Balam \& Yalavarthi, 2012; Randhawa et al., 2014; Sivajothi \& Reddy, 2016; Joshi et al., 2017). Corneal opacity develops as a result of stromal inflammation, tissue fibrosis, corneal epithelial thickening and vascularization (Morales et al., 2018).

The purpose of this case report is to put on records an unusual and rare case of corneal opacity secondary to microfilariosis due to $S$. labiatopapillosa in a water buffalo (Bubalus bubalis). This paper also describes in detail about the possible etiology behind corneal opacity in cattle and buffalo. This communication will help the clinicians to rule out parasitic aetiologies as a cause of corneal opacity in bovines.

\section{History and clinical observations}

A 15 year old pregnant water buffalo (Bubalus bubalis) was presented to RVP-TVCC, ICAR-Indian Veterinary Research Institute, Bareilly with the history of fever, anorexia, and corneal opacity for the past 10 days. On clinical examination, the animal was found to be lethargic, weak and anaemic. The buffalo also showed signs of visual impairment indicative of blindness. Physical parameters like respiratory rate and heart rate were found to be elevated.

The rectal temperature was within the normal range. The palpebral conjunctiva was found to be swollen and protruding, indicative of chemosis. Marked hyperaemia was also observed in the palpebral conjunctiva. The animal also showed extensive bilateral periocular oedema (Figure 1a). Ophthalmological examination identified marked bilateral corneal opacity that prevented the visualization of anterior chamber (Figure 1b). Haematological investigation revealed severe anaemia that was evident from the significantly lowered red blood cell count and haemoglobin concentration (Table 1). The blood picture was also suggestive of leukocytosis, neutrophilia, lymphocytopenia, and monocytosis.

Examination of wet film mount (peripheral blood) identified the presence of moving microfilaria (Figure 2). The prepared blood smear was stained using Giemsa stain and microscopic examination under oil immersion objective identified the presence of sheathed microfilaria. Based on morphological, the microfilariae was found to be belonging to Setaria sp. (Figure 3). Further morphometric analysis identified that the microfilaria had a length of $231 \mu \mathrm{m}$ without the sheath and $242 \mu \mathrm{m}$ with the sheath which is suggestive of S. labiatopapillosa microfilaria (Bino Sundar \& Ravindran, 2010). Peripheral blood smear examination was performed to rule out the presence of any haemoprotozoal infection. 

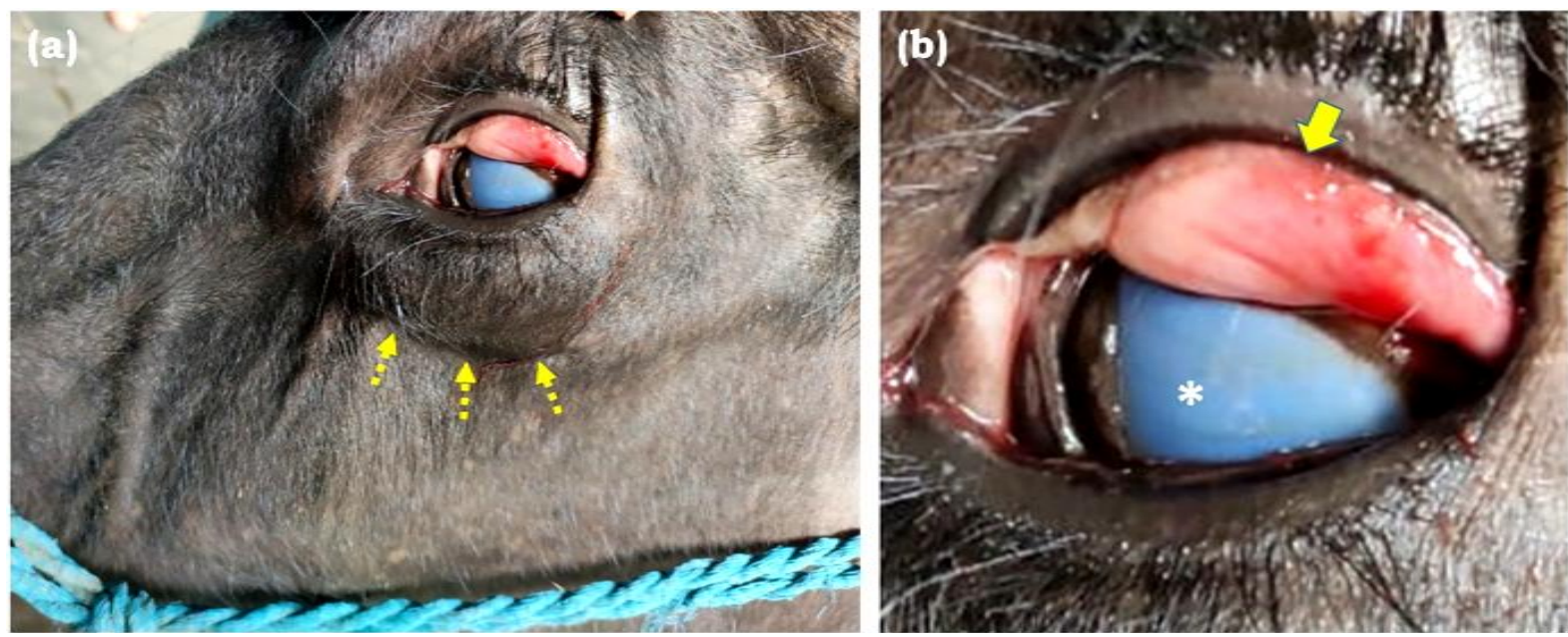

Figure 1 (a) Marked periocular edema (yellow arrow). (b) Chemosis characterized by swollen, hyperaemic and protruding palpebral conjunctiva (yellow arrow) and clearly visible corneal opacity that prevented visualization of anterior chamber of the eye (star).

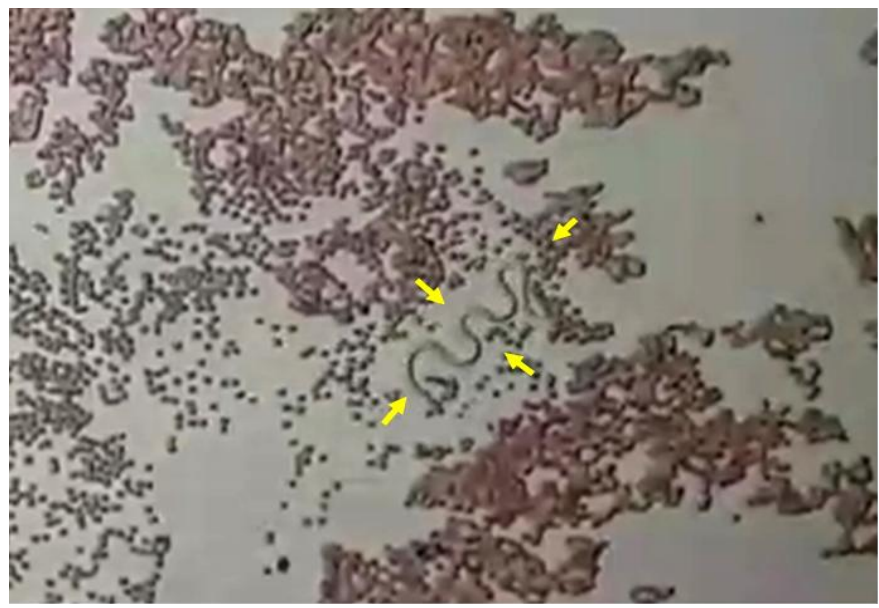

Figure 2 Wet film mount showing microfilaria - wet mount (10x)

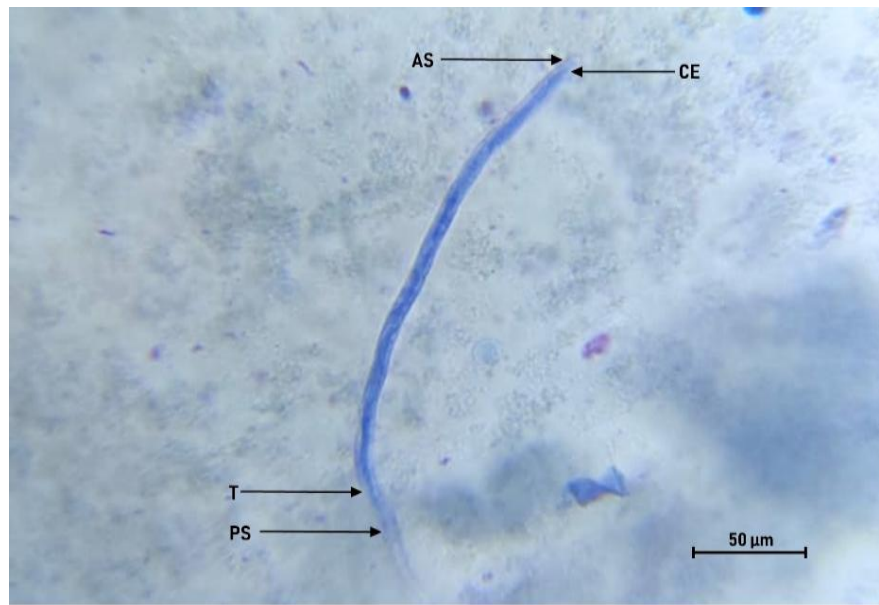

Figure 3 Presence of sheathed microfilaria suggestive of Setaria sp. in peripheral blood smear stained by Giemsa staining. (AS - Anterior sheath, CE - Cephalic end (head), T - Tail, PS - Posterior sheath)

Journal of Experimental Biology and Agricultural Sciences http://www.jebas.org 
Table 1 Hematological parameters of the buffalo on the day of the presentation

\begin{tabular}{|lcc|}
\hline Parameter & Value & Reference range $^{*}$ \\
\hline RBC count $\left(\times 10^{6} / \mu \mathrm{L}\right)$ & 1.62 & $5.0-10.0$ \\
\hline Hemoglobin $(\mathrm{g} / \mathrm{dL})$ & 4.2 & $8-15$ \\
\hline Total WBC count $\left(\times 10^{3} / \mu \mathrm{L}\right)$ & 26 & $4.0-12.0$ \\
\hline Differential leucocyte count & & \\
\hline Neutrophils $(\%)$ & 37 & $15-33$ \\
\hline Lymphocytes $(\%)$ & 40 & $45-75$ \\
\hline Monocytes $(\%)$ & 18 & $0-8$ \\
\hline Eosinophils $(\%)$ & 5 & $0-20$ \\
\hline Basophils $(\%)$ & 0 & $0-2$ \\
\hline
\end{tabular}

*Hematological reference ranges, The Merk Veterinary Manual - 11th edition (2016)

\section{Treatment}

The animal was treated with ivermectin at the dose of $200 \mu \mathrm{g} / \mathrm{kg}$ body weight subcutaneously on the day of presentation. Supportive therapy included intramuscular administration of injectable hematinic (Feritas, Intas Pharmaceuticals Ltd.). Unfortunately, the animal succumbed to death later that day before performing blood transfusion or any further investigation. Even though post-mortem examination was advised for further investigation, the owner declined the request.

\section{Discussion}

The bilateral corneal opacity was responsible for the visual impairment and blindness in the present case. Blindness occurs due to loss of corneal transparency. Morales et al. (2018) reported that blindness due to loss of corneal transparency can be successfully treated using ivermectin and topical cyclosporine (immunosuppressive agent) therapy, but may require several months of treatment. Hence, we have treated the animal with parenteral ivermectin. Microfilarial infection might have played an important role in the suppression of haemopoietic activity of the bone marrow (Rani et al., 2009). This may be responsible for the severely reduced red blood cell count and haemoglobin concentration. There was also a marked increase in the total leukocyte count which is indicative of infection.

The structural features of the identified microfilaria were in accordance with Bino Sundar \& Ravindran, (2010). The microfilariae of Setaria is sheathed with blunt anterior end and tapering posterior ends. The anterior sheath will be extending beyond the body hence has a prominent cephalic space. It also has a long and tapering tail that tapers gradually from anal pore to its tip. Similar to the anterior sheath the posterior/caudal sheath extends well beyond the tail tip (Bino Sundar \& Ravindran, 2010).
Histopathological investigations have identified free microfilariae in the corneas of canines affected with parasitic keratitis. Thus, corneal biopsy can be performed to identify the presence of free microfilaria in the corneal stroma (Morales et al., 2018). The presence of microfilaria in cornea can result in the destruction of collagen fiber and may result in varying degrees of inflammation. Histopathological examination of the cornea was not possible in the present case, such an investigation might have given an insight into the pathogenesis which would have helped us to confirm the etiology behind corneal opacity. Microfilariae has been also reported in the conjunctiva of equines naturally affected by Onchocerca $s p$ infection (Schmidt et al., 1982). But it was not associated with any distinguishing clinical abnormalities in horses. Onchocerca infection has been reported in dog, horses and cattle (Rommel et al., 2000; Sréter \& Széll, 2008). Onchocerca infection in dogs is manifested as either acute or chronic ocular disease. It is only seen in accidental hosts that are closely related to the natural host (Sréter \& Széll, 2008).

Corneal opacity has also been reported in cattle due to the accidental migration of Setaria digitata into the anterior chamber of eye with clinical signs such as corneal opacity, lacrimation, and blepharospasm (Mohan et al., 2009). The genus Thelazia (Spirurida, Thelaziidae) contains a group of eye worm spirurids that are responsible for ocular manifestations in domestic and wild animals. The presence of Thelazia $s p$. in the conjunctival sac of eye can also result in corneal opacity, conjunctivitis, and cloudiness. Thelazia $s p$. possesses a rough cuticle that causes irritation leading to the inflammation, ulceration, and finally perforation of the cornea (Balam \& Yalavarthi, 2012). Several protozoal parasites are also found to cause corneal opacity in bovines. Plasmodium bubalis infection was found to cause bilateral corneal opacity in buffaloes (Randhawa et al., 2014). Tropical theileriosis, a tick-borne disease of cattle and buffalo caused by the protozoa, Theileria annulata may also be associated with ocular signs like corneal opacity, bilateral conjunctivitis, severe lacrimation, bulging eyes, supraorbital oedema, photophobia, and impaired vision (Mahmmod et al., 2011; Joshi et al., 2017). The corneal opacity associated with tropical theileriosis is due to the infiltration of leucocytes into the cornea (Irvin \& Mwamachi, 1983). Another protozoan blood parasite, Trypanosoma evansi is also associated with corneal opacity in buffaloes (Sivajothi \& Reddy, 2016). Corneal opacity in buffalo can also be due to malignant catarrhal fever (MCF). MCF is caused by a closely related group of gammaherpes viruses. It is characterized by bilateral corneal opacity and keratitis in buffalo (Pfitzer et al., 2015). Infectious keratoconjunctivitis is another condition that causes corneal opacity, conjunctival hyperaemia, photophobia, and serious, mucoid and/or purulent ocular secretions in buffaloes. The most common etiological agents that are responsible for this condition include Moraxella sp., Escherichia coli, Corynebacterium sp., and Listeria sp. (Alsaad et al., 2012). 


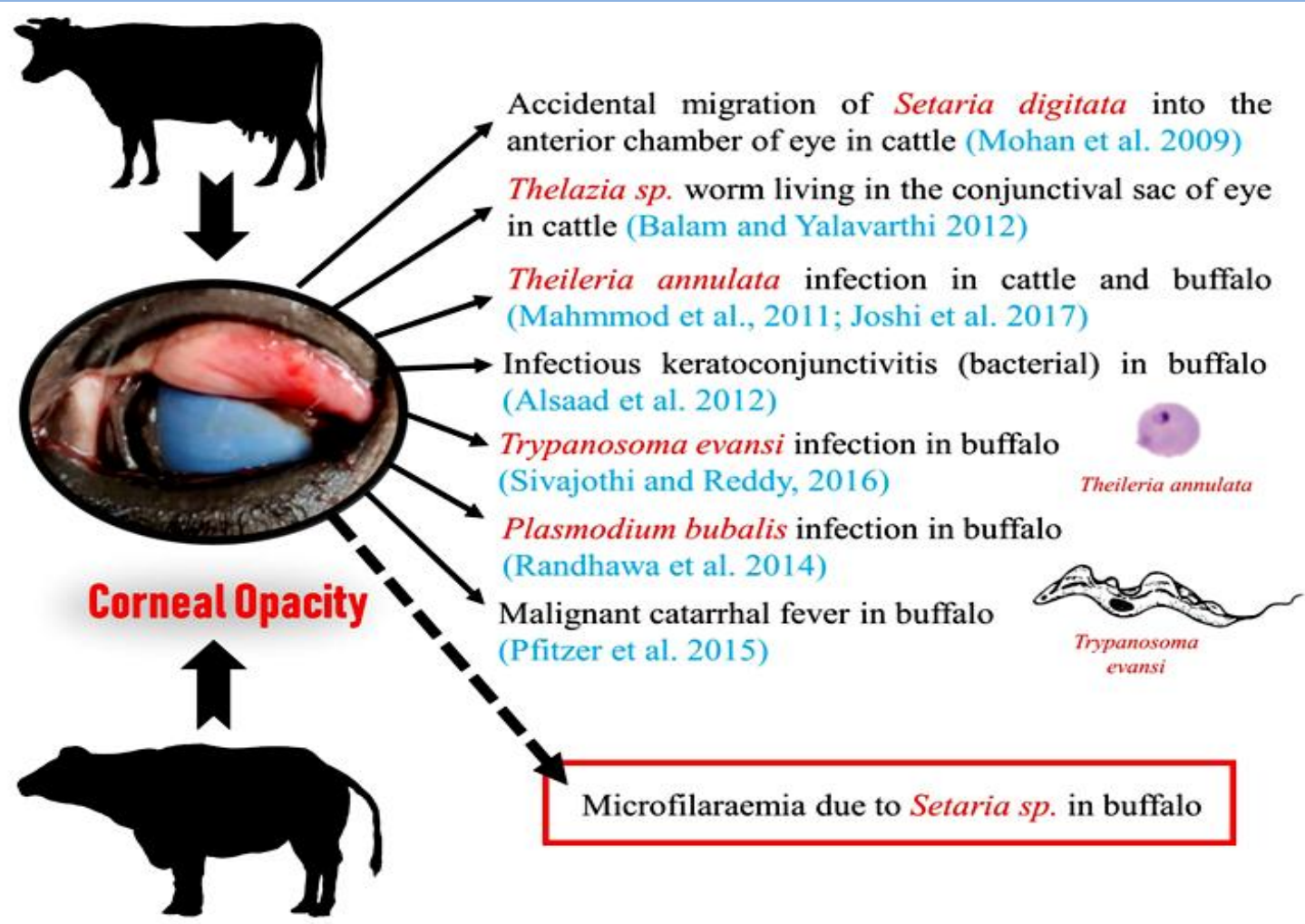

Figure 4 - Corneal opacity and the possible infectious and non-infectious aetiologies in cattle and buffaloes

Microfilariosis in buffalo has also been found to be associated with bilateral nasal bleeding (epistaxis), haemagalactia, and edema of the brisket region. Blood transfusion can be done to save the life of such animals suffering from severe anaemia (Rani et al., 2009). Even though epistaxis was not a clinical sign in the present case, due to the critically low hematological parameters blood transfusion was advised. But due to the poor condition and late presentation the buffalo collapsed before performing blood transfusion. Even though ivermectin and levamisole are considered to be the major therapeutic agents, ivermectin is the drug of choice that can be used for the treatment of microfilariosis. A single dose of subcutaneous injection of ivermectin at a dose rate of $200 \mu \mathrm{g} / \mathrm{kg}$ body weight is sufficient to clear the microfilariae from the circulation (Satish, 1996). Currently, long-acting preparations of ivermectin $(3.15 \% \mathrm{w} / \mathrm{v})$ are replacing the previously used formulations since they provide therapeutic concentration for a longer period (Sharun et al., 2019a; Sharun et al., 2019b). Herbal therapies are also evaluated for the management of microfilariosis. The extracts of Azadirachta indica leaves in different polar solvents were evaluated in vitro for their anthelmintic activity against the microfilariae of Setaria cervi. The result suggested that the methanolic and ethanolic extracts has anthelmintic effect which has to be further evaluated (Kausar, 2017).

\section{Conclusion}

Corneal opacity in cattle and buffalo is considered as a clinical manifestation that is seen associated with several infectious and non-infectious diseases. In such cases, identification of the etiological agent is important for its successful therapeutic management. The circumstantial evidence suggests that the corneal opacity associated with microfilaraemia in buffaloes can cause blindness due to the loss of corneal transparency. Microfilariosis can be accompanied by severe forms of anaemia that can be highly fatal if not corrected. The delayed presentation and severe nature of the disease lead to the death of buffalo in the present case. The proposed differential diagnosis chart (Figure 4) will be helpful in the diagnosis of cases presented with corneal opacity in bovines.

\section{Compliance with the ethical standards}

\section{Conflict of interest}

None of the authors has a financial or personal relationship with other people or organizations that could inappropriately influence or bias the content of the paper.

\section{Ethical approval}

This article does not contain any experimental studies with human or animal participants performed by any of the authors. All protocols followed were as per the guidelines from the standard textbooks in veterinary medicine/surgery and were ethical. 


\section{Funding}

None

\section{References}

Alsaad KM, Hussein SA, Mahmmoud EN (2012) Infectious kerato-conjunctivitis in local buffaloes breeds in Mosul, MOSULIRAQ. Basrah Journal of Veterinary Research 11: 148-154.

Balam D, Yalavarthi C (2012) Eye worm infection in a cattle-a case report. Veterinary World 5: 236-237.

Bino Sundar ST, Ravindran R (2010) Comparision of various methods for detection of microfilariae of Seteria in the blood of cattle. Tamilnadu Journal of Veterinary and Animal Sciences 6: 45-48.

Irvin AD, Mwamachi DM (1983) Clinical and diagnostic features of East Coast fever (Theileria parva) infection of cattle. The Veterinary Record 113 : 192-198.

Jayalakshmi J, Hafeez M, Kumar MU (2017) Prevalence of bovine microfilariasis in Rayalaseema region of Andhra Pradesh. Journal of parasitic diseases 41: 874-879.

Joshi V, Alam S, Dimri U, Bhanuprakash AG, Gopalakrishnan A, Ajith Y (2017) A rare case of Theileria annulata induced corneal opacity in a calf. Journal of Parasitic Diseases 41: 442-445.

Kausar S (2017) In vitro evaluation of antifilarial effect of Azadirachta indica leaves extract in different solvents on the microfilariae of Setaria cervi. Journal of parasitic diseases 41: 9-15.

Mahmmod YS, Elbalkemy FA, Klaas IC, Elmekkawy MF, Monazie AM (2011) Clinical and haematological study on water buffaloes (Bubalus bubalis) and crossbred cattle naturally infected with Theileria annulata in Sharkia province, Egypt. Ticks and tickborne diseases $2: 168-171$.

Mohan K, Ananda KJ, Shridhar NB, Puttalakshmamma GC, Placid E (2009) Corneal opacity due to Setaria digitata in a Jersey crossbred cow and its surgical management. Veterinary World $2: 69-70$.

Morales A, Perlmann E, Abelha AN, Levy CE, de Goes ACA, Safatle AM (2018) Keratitis due to microfilariae in dogs: a newly recognized disease. Veterinary Ophthalmology 21 : 305-311.

Pfitzer S, Last R, Espie I, Van Vuuren M (2015) Malignant Catarrhal Fever: An Emerging Disease in the African Buffalo (S yncerus caffer). Transboundary and Emerging Diseases 62: 288-294.

Randhawa SS, Singla LD, Dua K, Dewangan D, Singh D (2014) Treatment of bilateral corneal opacity caused by Plasmodium bubalis in a Murrah buffalo. Veterinary Practitioner 15: 136.

Rani NL, Sundar NS, Jayabal L, Devi VR (2009) Microfilariosis associated with epistaxsis in a she buffalo. Buffalo Bulletin 28: $171-172$.

Rommel M, Eckert J, Kutzer E, Korting W, Schnieder T (2000) Veterina r̈medizinische Parasitologie. Parey Verlag, Berlin.

Samatha V, Jyothisree CH, Babu KR (2016) Epidemiological and diagnostic studies of microfilariasis in buffaloes. Journal of Parasitic Diseases 40: 300-302.

Satish UD (1996) Clinico-therapeutic studies on microfilariosis in buffaloes (Bubalus bubalis). Doctoral dissertation, $\mathrm{PhD}$ thesis submitted to Andhra Pradesh Agricultural University, Hyderabad.

Schmidt GM, Krehbiel JD, Coley SC, Leid RW (1982) Equine ocular onchocerciasis: histopathologic study. American Journal of Veterinary Research 43 : 1371-1375.

Sharma MC, Joshi C (2002) Serum mineral and haematobiochemical profile of microfilariae infected cattle in India: Its effects on production and therapy. Asian-Australasian Journal of Animal Sciences 15 : 357-365.

Sharun K, Anjana S, Sidhique SA, Panikkassery S (2019a) Treatment of Sarcoptic mange infestation in rabbits with long acting injectable ivermectin. Journal of parasitic diseases 43(4): 733-736.

Sharun K, Shyamkumar TS, Aneesha VA, Dhama K, Pawde AM, Pal A. (2019b) Current therapeutic applications and pharmacokinetic modulations of ivermectin. Veterinary World 12(8): 1204-1211.

Sivajothi S, Reddy BS (2016) Polypeptide profiles of diminazene aceturate resistant Trypanosoma evansi organisms isolated from a Buffalo. Journal of Veterinary Science \& Medicine 4: 1-4.

Sréter T, Széll Z (2008) Onchocercosis: a newly recognized disease in dogs. Veterinary Parasitology 151: 1-13.

Sundar ST, D'Souza PE (2015) Morphological characterization of Setaria worms collected from cattle. Journal of Parasitic Diseases $39: 572-576$ 